\title{
Análise numérica da profundidade de submersão de um dispositivo coluna de água oscilante submetido à incidência de um espectro de ondas do tipo Pierson-Moskowitz
}

Numerical analysis of the Oscillating Water Column device submersion depth with a PiersonMoskowitz spectrum wave

\author{
M. J. Deus ${ }^{1 *}$; E. D. Santos ${ }^{2}$; L. A. Isoldi²; L. A. O. Rocha ${ }^{3}$; M. N. Gomes ${ }^{4}$ \\ ${ }^{1}$ Programa de Pós Graduação em Física e Astronomia, Universidade Tecnológica Federal do Paraná, 80230-901, \\ Curitiba-PR, Brasil \\ ${ }^{2}$ Universidade Federal do Rio Grande - FURG, 96203-900, Rio Grande-RS, Brasil \\ ${ }^{3}$ Universidade do Vale dos Sinos - UNISINOS, 93020-190, São Leopoldo-RS, Brasil \\ ${ }^{4}$ Instituto Federal do Paraná - Campus Paranaguá, 83215-750, Paranaguá-PR, Brasil
}

*deus@alunos.utfpr.edu.br

\begin{abstract}
Hoje em dia a utilização de energia no mundo é amplamente discutida, e, portanto é necessário que novas fontes renováveis de energia surjam. Uma fonte energética disponível é o oceano, onde a energia contida nas ondas do mar pode ser transformada em energia elétrica. Este trabalho tem o objetivo analisar numericamente o efeito da profundidade de submersão na potência hidropneumática disponível de um dispositivo conversor de energia das ondas do mar em energia elétrica, cujo princípio de funcionamento é o Coluna de Água Oscilante (CAO). Para representar o problema computacionalmente utilizou-se o modelo Volume of Fluid na interação fluido-estrutura entre a onda e o dispositivo CAO num tanque de ondas. Além disso, é considerado o espectro de Pierson-Moskowitz, que representa condições de mar. Neste estudo, as características do espectro de ondas foram: período significativo $T_{s}=7,50 \mathrm{~s}$, profundidade $h=10 \mathrm{~m}$ e altura significativa $H_{s}=1,50 \mathrm{~m}$. Os resultados indicam que a profundidade de submersão do dispositivo CAO, para as configurações geométricas e condições de mar adotadas neste trabalho, não tem influência significativa na potência hidropneumática disponível.

Palavras-chave: Coluna de Água Oscilante, Espectro de Ondas, Modelagem Computacional.
\end{abstract}

The discussion around energy and its uses is a current subject in the modern world, and the need for new and renewable sources of energy to appear is of a current issue. The ocean is a source of renewable energy available, where the kinetic ocean wave's energy can be converted into electrical energy. The goal of this paper is to perform an numerical analysis of the depth of a Oscillating Water Column (OWC) Device and its effects in the hydropneumatic power available inside the device. The Volume of Fluid model was employed to represent the problem inside the computer as a wave tank. Also, the Pierson-Moskowitz Wave Spectrum was used as a representation for the sea. The spectrum characteristics were: significant period $T_{s}$ $=7,50 \mathrm{~s}$, depth $h=10 \mathrm{~m}$ and significant height $H_{s}=1,50 \mathrm{~m}$. The results show that the submersion depth of the OWC Device (for the geometrical configuration and sea condition used) doesn't have domination for the hydropneumatic power. Insert the abstract here.

Keywords: Oscillating Water Column, Wave Spectrum, Computational Modeling

\section{INTRODUÇÃO}

Cada vez mais se faz necessário a utilização de energia ao redor do planeta. Para suprir essa necessidade, em geral são utilizados combustíveis fósseis [1]. A utilização de combustíveis fósseis contribui para a poluição e é prejudicial para a saúde humana, além de colaborar para a mudança climática e o efeito estufa. E, portanto, necessitam-se pesquisas a respeito de formas alternativas e renováveis de energia [2,3].

$\mathrm{O}$ uso da energia a partir de fontes renováveis têm se tornado relevante no contexto do desenvolvimento sustentável, visto que para realizar ações humanas de iluminação, aquecimento, transporte e comunicação, por exemplo; é necessário utilizar energia proveniente da natureza. Surge a necessidade de diminuir o impacto disso na natureza [4]. 
Uma possibilidade é a conversão da energia das ondas do mar em energia elétrica, visto que há diversas maneiras de se aproveitar a energia das ondas oceânicas. Uma das formas é através de um dispositivo cujo princípio de funcionamento é o de Coluna de Água Oscilante (CAO) [3].

Neste trabalho, o objetivo foi analisar o efeito da profundidade de submersão na potência hidropneumática disponível num dispositivo Coluna de Água Oscilante submetido a um espectro de ondas. As configurações geométricas dos dispositivos CAO analisados no presente trabalho foram definidos a partir de Gomes et al. (2018) [5].

Um tanque de ondas é utilizado para representar o problema de forma computacional. Para tanto é empregado o modelo multifásico Volume of Fluid (VOF) proposto por Hirt e Nichols (1981) [6], que é recomendado para representar a interação de dois fluidos imiscíveis, neste caso, água e ar.

$\mathrm{Na}$ literatura encontra-se uma variedade de trabalhos que utilizam o modelo Volume of Fluid (VOF) em simulações numéricas de conversão de energia das ondas do mar em elétrica [7, 8, 9, $10,11]$.

\section{REPRESENTAÇÃO DO PROBLEMA}

Uma das formas de energia contida no oceano é a das ondas, resultado do efeito do vento sobre a superfície da água se manifestado através de energia cinética. É possível aproveitar esse tipo de energia através de um dispositivo Coluna de Água Oscilante (CAO) [12], que são estruturas ocas dispostas parcialmente submersas com uma abertura para o mar abaixo da superfície livre da água, de acordo com o indicado na Figura 1.

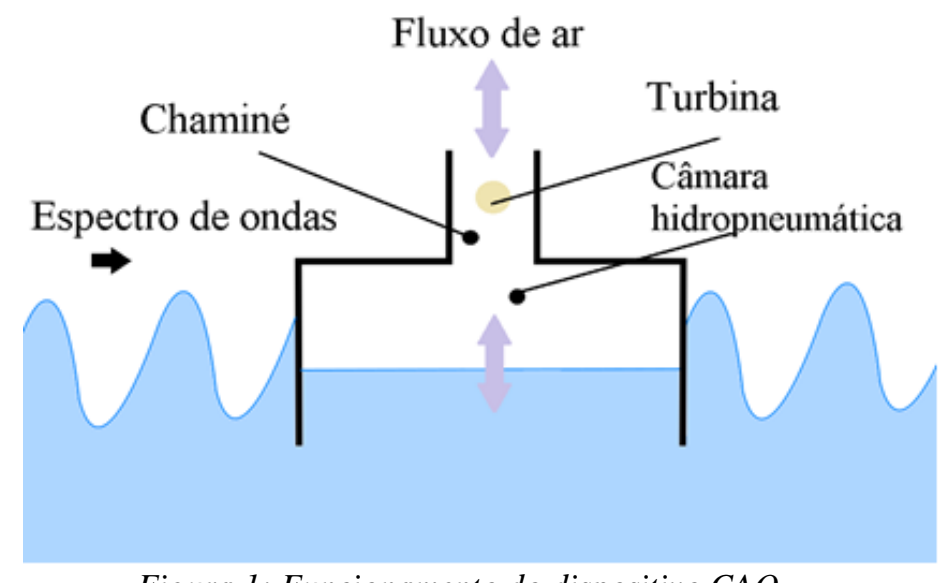

Figura 1: Funcionamento do dispositivo CAO.

A conversão em energia elétrica, de acordo com Cruz e Sarmento (2004) [12], ocorre de duas formas. Quando a onda atinge a estrutura, a pressão do ar dentro da câmara aumenta e ele é forçado a subir, passando pela chaminé e movimentando a turbina; e quando a onda volta para o oceano, o ar externo à chaminé retorna, devido à diminuição da pressão dentro da câmara, movimentando assim a turbina. Para manter o sentido de rotação constante é utilizado um tipo de turbina chamado Wells, que mantém seu sentido de rotação independente do sentido do fluxo de ar. Ligando a turbina a um gerador, ocorre a transformação de energia cinética das ondas em elétrica.

Uma das formas de representar a interação entre oceano e o dispositivo CAO é através de um tanque de ondas numérico. Neste trabalho é considerado um espectro de ondas de PiersonMoskowitz.

O espectro de ondas de Pierson-Moskowitz é representado por uma função de distribuição de energia que depende da altura significativa da onda $\left(H_{S}\right)$ e da frequência angular de pico $\left(\omega_{p}\right)$ [13]. Para o espectro de Pierson-Moskowitz, assume-se que a onda está em equilíbrio com o vento, onde a densidade espectral é: 


$$
S_{P M}(\omega)=\frac{5}{16} \frac{H_{s}^{2} \omega_{p}^{4}}{\omega^{5}} e^{-\left(\frac{5 \omega_{p}^{4}}{4 \omega^{5}}\right)}
$$

onde $H_{s}=1,5 \mathrm{~m}$ é a altura significativa, $\omega$ é a frequência da onda, em $\mathrm{rad} / \mathrm{s}$ e $\omega_{p}$ é a frequência de pico, em rad/s, que pode ser determinada por:

$$
\omega_{p}=\frac{2 \pi}{T_{s}}
$$

onde $T_{s}$ é o período significativo, em segundos, que nesse estudo foi adotado como $T_{s}=7,5 \mathrm{~s}$ [14].

O comprimento $\left(L_{T}\right)$ e a altura $\left(H_{T}\right)$ do tanque de ondas são determinados a partir do período significativo $\left(T_{S}\right)$, da altura significativa $\left(H_{S}\right)$ e da profundidade de propagação $(h)$ do espectro de ondas. Neste estudo, o espectro de ondas possui as seguintes características: $T_{s}=7,50 \mathrm{~s}, H_{s}=1,50$ $m, h=10 \mathrm{~m}$. Dessa forma, foi possível dimensionar o tanque de ondas apresentado na Figura 2.

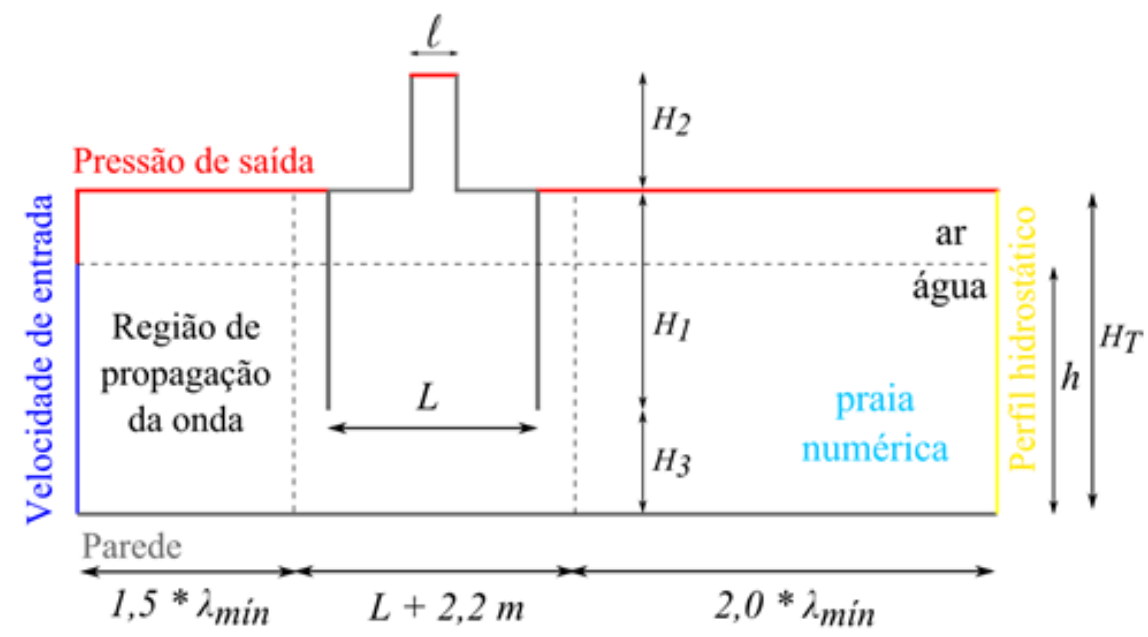

Figura 2: Representação esquemática do domínio computacional.

Nas partes superiores do tanque de ondas e da chaminé de saída do dispositivo CAO (linhas vermelhas na Figura 2), considera-se pressão atmosférica. Na parte inferior do tanque de ondas bem como nas paredes do dispositivo CAO é empregada a condição de não deslizamento e impermeabilidade com velocidade nula.

Na linha da direita (linha amarela na Figura 2), é adotado um perfil hidrostático como condição de contorno. Entre o dispositivo e essa linha está inserida uma praia numérica que tem como objetivo eliminar a reflexão das ondas dentro do tanque. A utilização do método da praia numérica consiste em adicionar termos de afundamento nas equações de momento em uma dada região do tanque. Esses termos são dados por Lisboa et al. (2016) [11] e Dizadji e Sajadian (2011) [15]:

$$
S=-\left[C_{1} \rho V+\frac{1}{2} C_{2} \rho|V| V\right]\left(1-\frac{z-z_{f s}}{z_{b}-z_{f s}}\right)
$$

onde $\mathrm{C}_{1}$ e $\mathrm{C}_{2}$ são os coeficientes de amortecimento linear e quadrático, respectivamente; $\rho$ é a massa específica do fluido; $V$ é a velocidade; $z$ é a posição normal; $z_{f s}$ é a posição vertical da superfície livre e $z_{b}$ a posição vertical do fundo; $x$ representa a posição horizontal, enquanto que 
$x_{s}$ marca o início e $x_{e}$ o fim da praia numérica, e $S$ é o momento de afundamento. Seguindo as recomendações de Lisboa et al. (2016) [11], $\mathrm{C}_{1}=20$ e $\mathrm{C}_{2}=0$.

Analiticamente, a elevação da superfície livre é calculada por:

$$
\zeta(t)=\sum_{n=1}^{n_{0}} \zeta_{a_{n}} \cos \left(k_{n} x-\omega_{n} t+\varepsilon_{n}\right)
$$

onde $(\zeta)$ representa a amplitude da componente da onda, $k_{n}$ é o número de onda da componente $(\mathrm{rad} / \mathrm{m}), x$ é a posição espacial $(\mathrm{m}), \omega_{p}$ é a frequência da onda, t é o tempo $(s), \varepsilon_{n}$ é o ângulo de fase randômico $(\mathrm{rad})$ e $n$ é a componente de cada onda [16]. Essa equação é idêntica à de uma onda regular, com exceção do ângulo de fase randômico, o que torna a onda irregular [16].

O número de onda pode ser calculado através da relação de dispersão, dada por Elangovan (2011) [17]:

$$
\omega^{2}=g k_{n} \tanh \left(k_{n} h\right)
$$

Como observa-se através da Figura 2, o gerador de ondas é posicionado do lado esquerdo do tanque. Numericamente, para a geração de ondas irregulares consideram-se as componentes de onda do espectro da Equação (5) e as componentes verticais $(w)$ e horizontais $(u)$ do espectro de ondas que são dadas por Chakrabarti (2005) [14] e Elangovan (2011) [17]:

$$
\begin{gathered}
u=\zeta_{a_{n}} g k_{n} \frac{\cosh \left(k_{n} z+k_{n} h\right)}{\omega_{n} \cosh \left(k_{n} h\right)} \cos \left(k_{n} x-\omega t\right) \\
w=\zeta_{a_{n}} g k_{n} \frac{\operatorname{senh}\left(k_{n} z+k_{n} h\right)}{\omega_{n} \operatorname{senh}\left(k_{n} h\right)} \operatorname{sen}\left(k_{n} x-\omega t\right)
\end{gathered}
$$

Inicialmente, no instante $t=0 \mathrm{~s}$, o tanque de ondas está ajustado com profundidade de 10 metros em todo o seu domínio.

Neste estudo, foram realizadas 21 simulações numéricas para verificar qual o efeito da profundidade de submersão do dispositivo CAO na potência hidropneumática disponível, analisando para tanto as geometrias apresentadas na Tabela 1 [5].

Tabela 1: Geometria do dispositivo CAO, segundo Gomes et al. (2018)[5].

\begin{tabular}{lcccc}
\hline & $H_{l}(m)$ & $H_{2}(m)$ & $L(m)$ & $l(m)$ \\
\hline$\lambda_{\max }=6,84 \mathrm{~m}$ & 1,5000 & 3,6320 & 6,8401 & 1,2107 \\
$\lambda_{\text {pico }}=65,35 \mathrm{~m}$ & 6,2003 & 11,2264 & 15,0898 & 3,7421 \\
$\lambda_{\min }=119,10 \mathrm{~m}$ & 6,2003 & 15,1556 & 28,8133 & 5,0519 \\
\hline
\end{tabular}

Como neste trabalho é considerado um espectro de ondas para que se possa estabelecer um referencial da geometria do dispositivo $\mathrm{CAO}$ e as ondas incidentes são considerados três situações: 1) O comprimento de onda mínimo $\left(\lambda_{\min }=119,10 \mathrm{~m}\right)$ referente a frequência mínima do espectro, 2) O comprimento da onda de pico $\left(\lambda_{\text {pico }}=65,35 \mathrm{~m}\right)$ referente a frequência de pico do espectro de ondas e 3$) \mathrm{O}$ comprimento de onda máximo $\left(\lambda_{\max }=6,84 \mathrm{~m}\right)$ referente a frequência máxima do espectro.

Na Tabela 1 são consideradas as configurações geométricas recomendadas em Gomes et al. (2018) [5] que conduzem a maior potência hidropneumática disponível me cada uma das três 
situações: $\lambda_{\max }=6,84 \mathrm{~m}, \lambda_{\text {pico }}=65,35 \mathrm{~m}$ e $\lambda_{\min }=119,10 \mathrm{~m}$, respectivamente. No presente trabalho foram analisadas as seguintes profundidades de submersão $(\mathrm{H} 3): 8,6 ; 8,8 ; 9,1 ; 9,4 ; 9,5 ; 9,7 \mathrm{e}$ $10,0 \mathrm{~m}$.

\subsection{MODELO VOLUME OF FLUID (VOF)}

No modelo VOF, as equações de conservação da massa, momento e transporte de fração volumétrica são empregadas quando há dois fluidos imiscíveis, ar e água neste caso. Para resolvêlas, utiliza-se o Método dos Volumes Finitos [18]. A equação da conservação da massa é dada por:

$$
\frac{\partial \rho}{\partial t}+\nabla \cdot(\rho \cdot \vec{v})=0
$$

onde $\rho$ é a densidade da mistura $\left(\mathrm{kg} / \mathrm{m}^{3}\right)$ e $\vec{v}$ é o vetor velocidade do fluxo $(\mathrm{m} / \mathrm{s})$

A equação da conservação do momento é:

$$
\frac{\partial(\rho \vec{v})}{\partial t}+\nabla \cdot(\rho \vec{v} \vec{v})=-\nabla p+\nabla(\tau)+\rho g
$$

onde $p$ é a pressão $(P a)$ e $\tau$ é o tensor de deformação $\left(N / m^{2}\right)$.

O método VOF é empregado quando há a interação entre dois ou mais fluidos imiscíveis, o que significa dizer que uma fase não pode ocupar a outra [6, 19].

Neste estudo, as frações de volume arepresentam as fases dentro do problema. Se a célula possui água, $\alpha_{\text {agua }}=1$ e $\alpha_{a r}=1$ - $\alpha_{\text {agua }}$. Assim, a equação para as frações de volumes é:

$$
\frac{\partial(\rho \alpha)}{\partial t}+\nabla \cdot(\rho \cdot \alpha \vec{v})=0
$$

As densidades e viscosidade da mistura são dadas por:

$$
\begin{aligned}
& \rho=\alpha_{\text {água }} \rho_{\text {água }}+\alpha_{a r} \rho_{a r} \\
& \mu=\alpha_{\text {água }} \mu_{\text {água }}+\alpha_{\text {ar }} \mu_{a r}
\end{aligned}
$$

Para a solução numérica dessas equações utilizou-se o método PRESTO para discretizar o momento e a pressão. Para o acoplamento pressão-velocidade, foi empregado o método PISO, enquanto que o GEO-RECONSTRUCTION foi utilizado na fração volumétrica.

\section{RESULTADOS}

Considerando as dimensões apresentadas na Tabela 1, 21 simulações (considerando a profundidade de submersão $H_{3}$ igual a: 8,$6 ; 8,8 ; 9,1 ; 9,4 ; 9,5 ; 9,7$ e $10,0 \mathrm{~m}$ ) foram realizadas a fim de verificar qual o efeito da profundidade de submersão do dispositivo CAO na potência hidropneumática disponível.

Para calcular os valores médios de vazão mássica, pressão interna da câmara e potência hidropneumática disponível, foi empregada a média transiente RMS (Root Mean Square). O cálculo para a média $R M S$ de uma variável arbitrária é dada pela Equação 13. 


$$
x_{R M S}=\sqrt{\frac{1}{N} \sum_{i=1}^{N} x_{i}{ }^{2}}
$$

Na Figura 3 é apresentado o comportamento qualitativo da média $R M S$ da vazão mássica em função da profundidade de submersão $\left(H_{3}\right)$ para os três conjuntos de análise. Na Tabela 2 são apresentados os valores da média $R M S$ para cada um dos casos apresentados na Figura 3.

A medida de vazão mássica representa a quantidade média de ar que está sendo movimentado dentro da câmara durante a simulação. É interessante notar, tanto da Figura 3 quanto da Tabela 2, que a profundidade de submersão $\left(H_{3}\right)$ não interfere significativamente termos de vazão mássica, mantendo-se praticamente constante em relação ao comprimento de onda em questão.

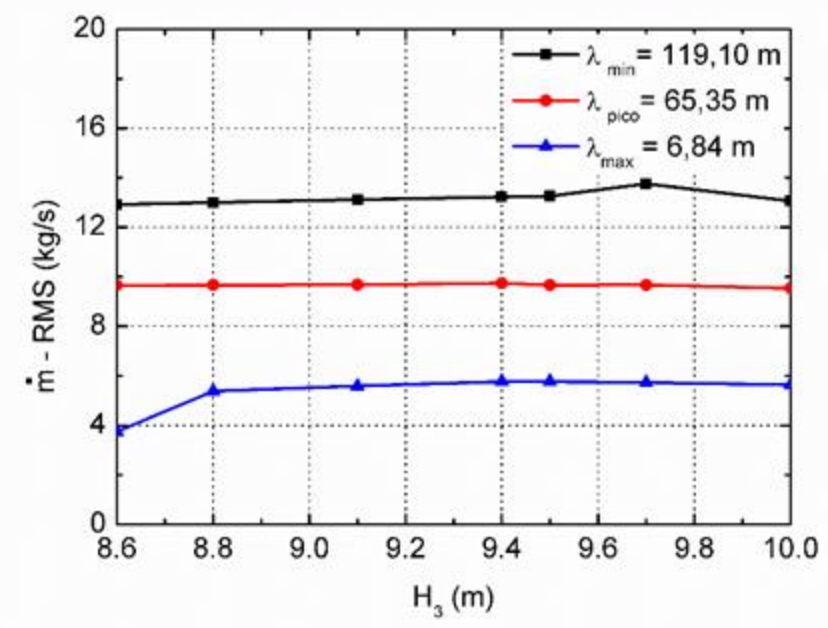

Figura 3: Vazão mássica em função da profundidade de submersão.

Uma análise semelhante é realizada para o comportamento da pressão, como o indicado na Figura 4. Os resultados obtidos para a média $R M S$ da pressão dentro da câmara em função da profundidade de submersão $\left(H_{3}\right)$ para os diferentes comprimentos de onda indicam que a medida que o dispositivo está mais submerso a pressão média $R M S$ tende a diminuir. Em algumas situações a variação média da pressão é significativa como, por exemplo, para $\lambda_{\max }=6,84 \mathrm{~m}$ ocorre uma diferença média de cerca de $10 \mathrm{~Pa}$ dependendo da profundidade de submersão $\left(\mathrm{H}_{3}\right)$, vide Tabela 2. 
Tabela 2: Valores médios RMS para vazão mássica, pressão e potência hidropneumática considerando a variação da profundidade de submersão do dispositivo CAO.

\begin{tabular}{|c|c|c|c|c|}
\hline$\lambda(\mathbf{m})$ & $\mathrm{H}_{3}(\mathrm{~m})$ & $\dot{\mathbf{m}}(\mathrm{kg} / \mathrm{s})$ & $\mathbf{p}(\mathbf{P a})$ & $\mathbf{P}_{\text {hid }}(\mathbf{w})$ \\
\hline 6,84 & 8,6000 & 3,7535 & 5,5980 & 81,2292 \\
\hline 6,84 & 8,8000 & 5,3862 & 7,2166 & 95,9240 \\
\hline 6,84 & 9,1000 & 5,5909 & 11,2843 & 36,9799 \\
\hline 6,84 & 9,4000 & 5,7684 & 14,6047 & 44,0696 \\
\hline 6,84 & 9,5000 & 5,7684 & 14,0034 & 43,7460 \\
\hline 6,84 & 9,7000 & 5,7289 & 13,7006 & 42,2375 \\
\hline 6,84 & 10,0000 & 5,6350 & 14,7937 & 41,2646 \\
\hline 65,35 & 8,6000 & 9,6502 & 13,3290 & 1918,7856 \\
\hline 65,35 & 8,8000 & 9,6636 & 13,9202 & 1936,0437 \\
\hline 65,35 & 9,1000 & 9,6751 & 14,9776 & 2011,8142 \\
\hline 65,35 & 9,4000 & 9,7408 & 16,2672 & 2172,9153 \\
\hline 65,35 & 9,5000 & 9,6644 & 16,2393 & 2074,1131 \\
\hline 65,35 & 9,7000 & 9,6667 & 16,6221 & 2114,8774 \\
\hline 65,35 & 10,0000 & 9,5337 & 17,4622 & 2091,5388 \\
\hline 119,10 & 8,6000 & 12,9155 & 15,0100 & 8330,9214 \\
\hline 119,10 & 8,8000 & 12,9928 & 15,5001 & 8504,0505 \\
\hline 119,10 & 9,1000 & 13,1164 & 16,2362 & 8793,0190 \\
\hline 119,10 & 9,4000 & 13,2204 & 17,1471 & 9064,0831 \\
\hline 119,10 & 9,5000 & 13,2526 & 17,8240 & 9152,5975 \\
\hline 119,10 & 9,7000 & 13,7542 & 18,6114 & 9634,2127 \\
\hline 119,10 & 10,0000 & 13,0602 & 18,5157 & 8208,5433 \\
\hline
\end{tabular}

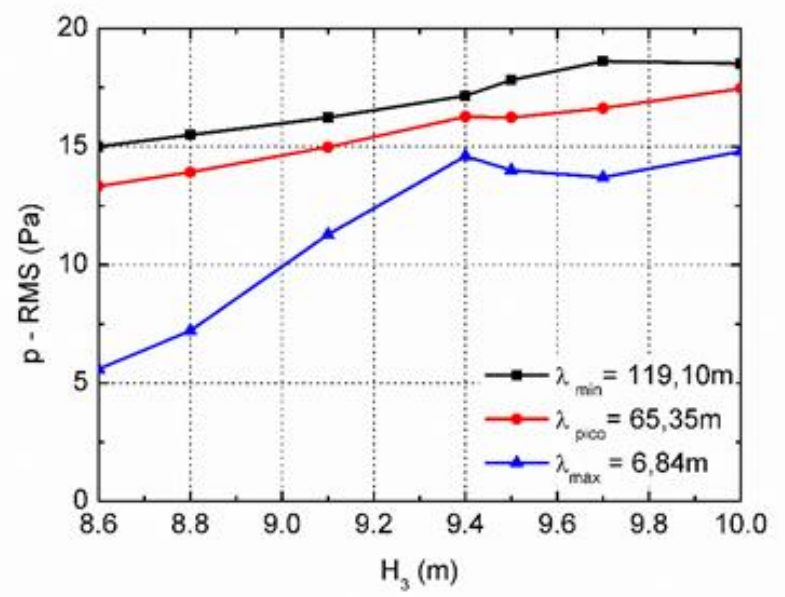

Figura 4: Pressão em função da profundidade de submersão.

Conhecendo-se a vazão mássica e a pressão determina-se a potência hidropneumática segundo a expressão [15]:

$$
P_{\text {hid }}=\left(p_{a r}+\frac{\rho_{a r} v_{a r}^{2}}{2}\right) \frac{\dot{\mathrm{m}}}{\rho_{a r}}
$$

onde: $P_{a r}$ é a pressão estática na chaminé do dispositivo $\mathrm{CAO}(\mathrm{Pa}) ; \rho_{a r}$ é a massa específica do ar $\left(\mathrm{kg} / \mathrm{m}^{3}\right)$; $\dot{\mathrm{m}}$ corresponde a vazão mássica de ar no duto da turbina $(\mathrm{kg} / \mathrm{s})$ e $v_{a r}$ refere-se a velocidade do ar no duto da turbina $(\mathrm{m} / \mathrm{s})$, que pode ser dada por: 


$$
v_{a r}=\frac{\dot{m}}{A \rho_{a r}}
$$

sendo $A$ a área da secção transversal da chaminé $\left(m^{2}\right)$.

Na Figura 5 e na Tabela 2, observa-se que a profundidade de submersão $\left(H_{3}\right)$ não tem efeito significativo na potência hidropneumática disponível. Isso ocorre devido ao fato de que a vazão mássica é predominante na avaliação da potência hidropneumática, conforme constatado na Equação 14. E nesta situação a vazão mássica pouco se altera com a variação da profundidade de submersão considerada neste trabalho.

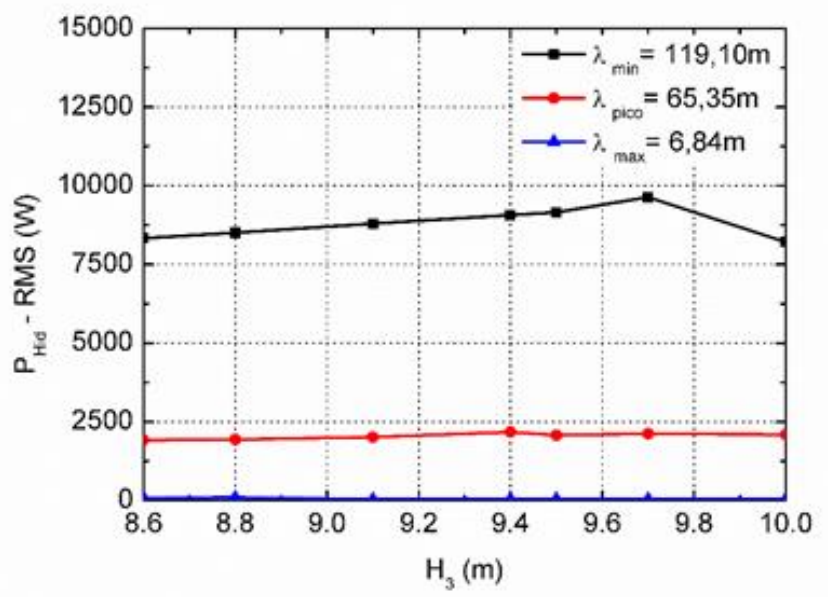

Figura 5: Potência hidropneumática em função da profundidade de submersão.

Nota-se ainda, da Tabela 2, que as maiores diferenças médias para vazão mássica, pressão e potência hidropneumática ocorreram para as câmaras CAO com configurações geométricas menores. No entanto, para os dispositivos com configuração geométrica maior, a diferença não é significativa, onde houve uma diferença de 1,15 vezes entre o melhor e o pior caso.

\section{CONCLUSÃO}

No presente trabalho foi realizada uma análise numérica da influência da profundidade de submersão de um dispositivo conversor de energia das ondas do mar do tipo CAO submetido a um espectro de ondas do tipo Pierson-Moskowitz.

Os resultados indicam que a profundidade de submersão tem influência na pressão dentro da câmara hidropneumática. No entanto, a quantidade de ar que está se movimentando pela chaminé e a potência hidropneumática não sofrem alterações tão significativas, permanecendo praticamente constantes durante todos os casos estudados. Isso ocorre devido ao fato de que a vazão mássica é predominante no cálculo da potência hidropneumática.

\section{AGRADECIMENTOS}

Os autores agradecem ao Instituto Federal do Paraná (IFPR), a Universidade Federal do Rio Grande (FURG), a Universidade Federal do Rio Grande do Sul (UFRGS), a Universidade do Vale do Rio dos Sinos (Unisinos), a Fundação Araucária, a Fundação de Amparo à Pesquisa do estado do Rio Grande do Sul (FAPERGS) e ao Conselho Nacional de Desenvolvimento Cientifico e Tecnológico (CNPq). 


\section{REFERÊNCIAS BIBLIOGRÁFICAS}

1. Schlager N, Weisblatt. Alternative Energy. Thomsom Gale, EUA, 200

2. Gozgor G, Lau CKM, Lu Z. Energy consuption and economic growth: new evidence from OECD countries. Energy, Elsevier 2018;153:27-34. doi: 10.1016/j.energy.2018.03.158

3. Kaygusuz K. Energy for suitable development: key issues and challenges. Energy sources B Energ Econ Plann. 2007;2(1):73-83.doi:10.1080/15567240500402560

4. Twindell J, Weir T. Renew Energ Res. Taylor and Francis. London. 2006.

5. Gomes MN, Deus MJ, Santos ED, Isoldi LA, Rocha LAO. Analysis of the geometric contraints employed in Constructal Design for Oscillating Water Column devices submitted to the wave spectrum through a numerical approach. 2018, 14Th International Conference of Difusion in Solids and Liquids, Amsterdam.

6. Hirt CW, Nichols BD. Volume of Fluid (VOF) method for the dynamics of free boundaries. J Comput Phys. 1981;39(1):201-225. doi.org/10.1016/0021-9991(81)90145-5

7. Horko MM. CFD Optimization of an oscillating water column energy converter. Msc Thesis in Engineering Science, School of Mechanical Engineering. The University of Western, Western, 2007.

8. Conde JMP, Gato LMC. Numerical study of the air-flow in an oscillating water column wave energy converter. Renew energ. 2008;33:2637-2644. doi.org/10.1016/j.renene.2008.02.028

9. Marjani AE, Ruiz FC, Rodriguez MA, Santos MTP. Numerical modelling in wave energy conversion systems. Energ. 2008;33:1246-1256. doi: 10.1016/j.energy.2008.02.018

10. Gomes MN. Constructal Design de dispositivos conversores de energia das ondas do mar em energia elétrica do tipo Coluna de Água Oscilante. [tese], Universidade Federal do Rio Grande do Sul, Brasil, 2014.

11. Lisboa RC, Teixeira PRF, Didier E. Simulação de propagação de ondas regulares e irregulares em um canal bidimensional com praia numérica. VII Seminário e Workshop em Engenharia Oceânica. Rio Grande, 2016.

12. Cruz J, Sarmento A. Wave Energy - Introdução aos aspectos tecnológicos, econômicos e ambientais. Institute of Ambient. Alfragide, 2004.

13. Ansys. Theory Guide FLUENT 2016, 2016.

14. Chakrabarti SK. Handbook of Offshore Engineering. Elsevier, Amsterdam, London, 2005

15. Dizadji N, Sajadian SE. Modeling and optimization of the chamber of OWC system, Energ. 2011;36:2360-2366. doi: 1016/j.energy.2011.01.010

16. Dean RG, Dalrymple RA. Water wave mechanics for engineering and scientists. World scientific, Singapore, 1991

17. Elangovan M. Simulation of irregular waves by CFD. World Academy of Science. Engin Technol. 2011;5:427-431.

18. Veersteg HK, Malalasekera W. An introduction do Computational Fluid Dynamics. Malasya: Pearson, 2007.

19. Lv X, Zou Q, Reeve D. Numerical simulation of overflow at vertical weirs using a hybrid level set/Vof method. Adv Water Resour. 2011;1320-1334. doi: 10.1016/j.advwatres.2011.06.009 